\title{
CAD Design of Disc Cam Based on MATLAB and Solidworks
}

\author{
Mingxia Zhao \\ Baoji Vocational and Technical College, Baoji Shaanxi, 721013, China
}

Keywords: Cam profile, MATLAB, Solidwoks.

\begin{abstract}
The cam mechanism is widely used in the field of mechanical engineering thanks to its small structure and wide variety of motion characteristics. The cam, as the non-standard article, has many kinds of uses, it's a big, long and inefficient work when designing to be used in different occasions separately. On the basis of the development of 3D CAD/CAM technology, the cam design and CAD technology are combined to develop the corresponding cam design CAD software, which can reduce the repetitive labor in the design, speed up the development speed and improve the design quality.
\end{abstract}

\section{Introduction}

This paper mainly studies the disc-based cam CAD design method based on Solidworks in which the cam profile curve fitting to produce a smooth curve profile by using MATLAB powerful data processing function and the preparation of the program. The SolidWorks is choosed as the platform to import the two-dimensional contour curve into SolidWorks, so the three-dimensional model of CAM mechanism is set up. After that, SolidWorks is developed two times with VB as the Language tool to realize the parametric modeling of CAM mechanism and improve the design efficiency of CAM.

Cam mechanism, as a high auxiliary movement, has become one of the typical common institutions because it can complete any established movement law and combine with other institutions properly in use to meet various complex movement requirements of the mechanical movement in accordance with the movement requirements of the moving parts. Thanks to its good stability, movement characteristics and reliability, fewer components, small size, etc., it gets widely used in mechanical equipment. However, given the fact that CAM is more complex and it is required to have a high precision of moving parts in some occasions, it's difficult to be used in the design and processing. The design of the disc cam mechanism generally includes: 1 . The cam type and the choice of the moving motion of the moving parts; 2 . The determination of the basic parameters; 3 . The design of the three-dimensional profile; 4 . The analysis of the motion performance. In the first part of this paper, the basic theory of disc cam is studied, the mathematical model is established, and the cam contour curve is obtained by MATLAB. In the second part, it carries on the parametric three-dimensional modeling according to the cam contour coordinate value in the SolidWorks, later uses VB to carry on the interface development and the data programming and obtain the interface simple cam design plug-in. The user can automatically generate the cam three-dimensional model as long as they input parameter.

\section{MATLAB Cam Profile Curve Design}

The contour curve of the cam is actually composed of several sections. The contour curve is segmented and expressed in the form of an equation under the premise of the regular motion of the moving parts. The two-dimensional contour curve and the motion line of the cam can be generated by programming the function expression in MATLAB.

\section{MATLAB cam profile curve design method}

The analytic design of cam contour with MATLAB can reduce the workload greatly, and the different types of cam contour can be designed by designing different modules. The design process of MATLAB consists of main program and subroutine. The main program carries out the input of the 
parameter and the output of the subroutine and the result. The subroutine is divided into two parts including the selection of the moving law of the follower and the coordinate of the contour curve and the calculation of the pressure angle. The coordinates of the point on the cam contour curve can be realized by the independent function, programming for the different function modules, drawing the contour curve and displacement of the cam with the drawing tool of MATLAB and the speed acceleration diagram line [1], the process is shown in Fig. 1.

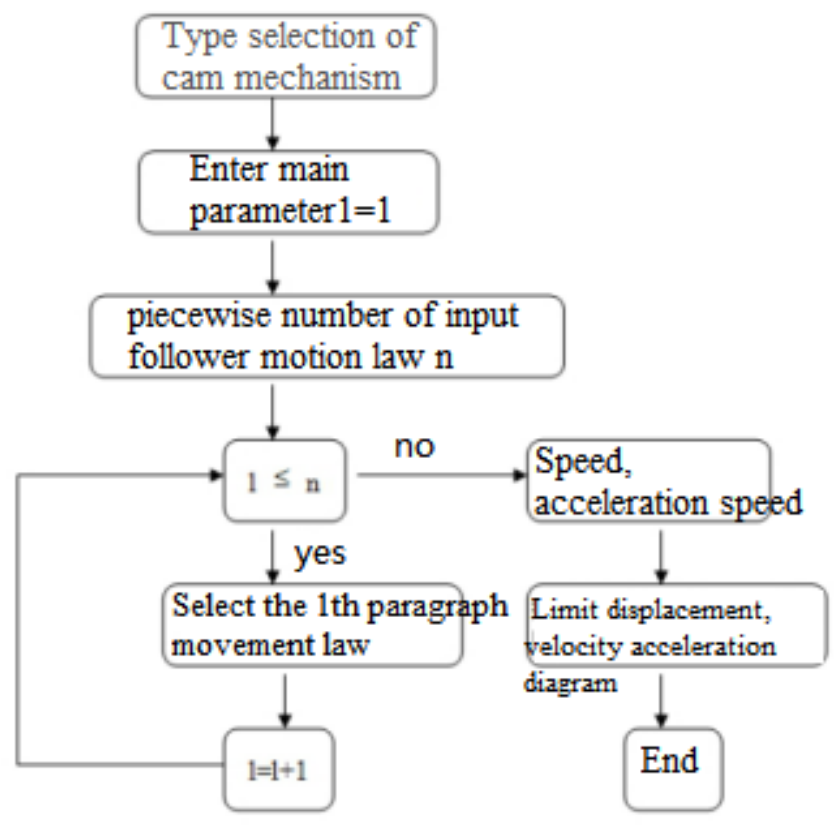

Fig. 1 MATLAB Workflow Diagram

\section{Round contour programming}

When using MATLAB to design the cam, it should be noted: First, select the appropriate cam type according to the movement requirements of the body; Second, select the moving motion of the follower, and derive the equations of the movement phase; Third: determine the cam base size. Fourth, determine the contour curve of the cam[2].

Take the roller cam as an example. Given the known conditions: design a pair of straight-headed roller cam mechanism, the moving process of the follower is required to be rise-stop-down-stop. When the cam turns over 1500 , the follower is raised by sine acceleration by $40 \mathrm{~mm}$. The cam rotation angle is 600 , the return stage cam angle 900 , the follower comes back to the origin sine acceleration speed. When the cam turns over the remaining 600, the follower is in the return stage.

Determine the equation and parameters

(1) The moving equation of the follower

$\varphi$ is set to be the angle of the cam for the stroke. $\varphi^{\prime}$ is the return angle of the cam for the return stroke, and the motion law of sinusoidal acceleration is in the process of pushing

The deduction equation is: $\left(0 \leq \varphi \leq 150^{\circ}\right)$

$s=h[\varphi / \phi-(1 / 2 \pi) \sin (2 \pi p / \phi)]$

$v=(h \omega / \phi)[1-\cos (2 \pi \varphi / \phi)]$

$a=\left(2 \pi h \omega^{2} / \phi^{2}\right) \sin (2 \pi p / \phi)$

Far stop: $\left(150^{\circ} \leq \varphi \leq 210^{\circ}\right)$

$s=h, a=0, v=0$,

Back to the sine acceleration movement law, the return equation is: $\left(210^{\circ} \leq \varphi^{\prime} \leq 300^{\circ}\right)$

$$
\begin{aligned}
& s=h\left[1-\varphi^{\prime} / \phi^{\prime}+(1 / 2 \pi) \sin \left(2 \pi \varphi^{\prime} / \phi\right)\right] \\
& v=\left(h \omega / \phi^{\prime}\right)\left[1-\cos \left(2 \pi \varphi^{\prime} / \phi^{\prime}\right)\right] \\
& a=-\left(2 \pi h \omega^{2} / \phi^{\prime 2}\right) \sin \left(2 \pi p^{\prime} / \phi^{\prime}\right)
\end{aligned}
$$


Near halt: $\left(300^{\circ} \leq \varphi \leq 360^{\circ}\right)$

$s=0, a=0, v=0$

Determination of radius of base circle and roller radius

The push process is sine. As it's troublesome to calculate the base circle and roller radius according to Formula 1.1 and 1.2 manually, MATLAB is adopted to write M file and calculate it.

$\%$ Filename:tulunshj1.m

$\mathrm{t} 0=\operatorname{input}(\operatorname{delta} 0=150)$;

$\mathrm{t}=\operatorname{input}(\operatorname{delta}=150)$;

$\mathrm{e}=\operatorname{input}(\mathrm{e}=0)$;

$\mathrm{h}=\operatorname{input}(\mathrm{h}=40)$;

$\mathrm{s}=\mathrm{h} *(\mathrm{t} / \mathrm{t} 0-\sin (2 * \mathrm{pi} * \mathrm{t} / \mathrm{t} 0) /(2 * \mathrm{pi})) ;$ Movement law (omitted)

$\mathrm{y}=(\mathrm{s} 0+\mathrm{s}) \cdot{ }^{*} \cos (\mathrm{t})+\mathrm{e} \cdot{ }^{*} \sin (\mathrm{t})$; cam theory contour curve equation

$\mathrm{dx}=(\mathrm{ds}-\mathrm{e}) \cdot * \sin (\mathrm{t})+(\mathrm{s} 0+\mathrm{s}) \cdot * \cos (\mathrm{t})$ :

$\mathrm{dy}=(\mathrm{ds}-\mathrm{e}) \cdot * \cos (\mathrm{t})-(\mathrm{s} 0+\mathrm{s}) \cdot * \sin (\mathrm{t})$

$\mathrm{ddx}=(\mathrm{dds}-\mathrm{s} 0-\mathrm{s}) \cdot * \sin (\mathrm{t})+(2 * \mathrm{ds}-\mathrm{e}) \cdot * \cos (\mathrm{t})$;

$\mathrm{ddy}=(\mathrm{dds}-\mathrm{s} 0-\mathrm{s}) \cdot * \cos (\mathrm{t})-(2 * \mathrm{ds}-\mathrm{e}) \cdot * \sin (\mathrm{t})$;

$\mathrm{rh} 0=(\mathrm{dx}, \wedge 2+\mathrm{dy} . \wedge 2) .^{\wedge} 1.5 . / \mathrm{abs}\left(\mathrm{ddy} .{ }^{*} \mathrm{dx}-\mathrm{ddx} . * \mathrm{dy}\right) ; \%$ cam theory contour curvature radius

$\mathrm{rrm}=\min (\mathrm{rh} 0) ; \%$ cam theoretical contour radius of curvature minimum

The minimum value of $\mathrm{R} 0$ is obtained by running the program, the $\mathrm{r} 0=30 \mathrm{~mm}$ is determined according to the actual situation, and finally, the RR is $10 \mathrm{~mm}$.

Compile contour curve $M$ file

The cam rotates one cycle, according to the movement requirements of the four stages of the follower, defines $\pi$ / pi of the matrix step length, $\mathrm{r} 0$ is the base circle radius, $\mathrm{rr}$ is the roller radius, $\mathrm{e}$ is the offset, $\mathrm{j} 1, \mathrm{j} 2, \mathrm{j} 3, \mathrm{j} 4$ are the angles of the cam in the four movement process. After MATLAB program and input parameters are designed, the program will draw the cam contour, stimulate the cam movement, and thus generate the moving line of a follower. The procedure is as follows:

Clear all;

Close all;

Clc

$\mathrm{J}=0: 3: 360$ (omitted)

$\%$ Sine acceleration motion regular return section, cam angle $\mathrm{j} 3-\mathrm{j} 2$.

$$
\begin{aligned}
& s(i)=h *(1-(j(i)-j 2) /(j 3-j 2)+\sin (2 * \text { pi } *(j(i)-j 2) /(j 3-j 2)) /(2 * p i)) ; \\
& v(i)=h * w^{*}\left(\cos \left(2 * p^{*} *(j(i)-j 2) /(j 3-j 2)\right)-1\right) /(j 3-j 2) ; \\
& a(i)=-2 * p i * h^{*} w^{\wedge} 2 * \sin (2 * p i *(j(i)-j 2) /(j 3-j 2)) /((j 3-j 2) \wedge 2) ;
\end{aligned}
$$

Else $\%$ near the stop, the cam angle is $360-\mathrm{j} 3$.

$$
\begin{aligned}
& \mathrm{S}(\mathrm{i})=0 ; \\
& \mathrm{V}(\mathrm{i})=0 ; \\
& \mathrm{A}(\mathrm{i})=0 ;
\end{aligned}
$$

end

$\mathrm{t}=$ pi / 180;

If you select the curve of other motion laws, likewise, you can further obtain contour curve after obtaining the curve. The procedure is as follows:

$\%$ Draw the cam theory profile, the actual profile

$\mathrm{r} 0=30$;

$\mathrm{e}=0$;

$\mathrm{rr}=10$

$\mathrm{s} 0=\operatorname{sqrt}\left(\mathrm{r} 0 \wedge 2-\mathrm{e}^{\wedge} 2\right) ;$

$\mathrm{X}=\operatorname{rand}(1,121)$;

$\mathrm{Y}=\operatorname{rand}(1,121)$;

$\mathrm{Xa}=\operatorname{rand}(1,121)$; 


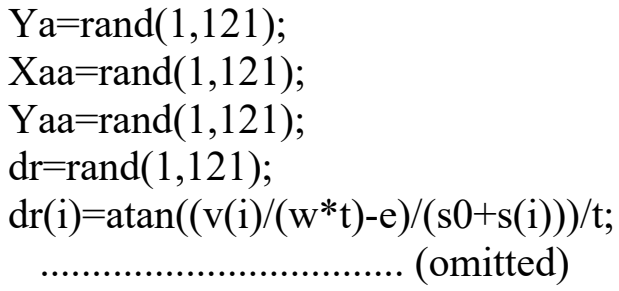

Legend ('base circle', 'actual profile')

\section{Contour curve file operation}

While running the prepared $\mathrm{M}$ file, you can get the desired results. The operation is as follows: open the "file" in the MATLAB interface to retrieve the M file and then point 㥓 to run the program, The contour curve of the cam and the follower displacement, speed, acceleration speed will be generated in turn, as shown in Fig. 2 .

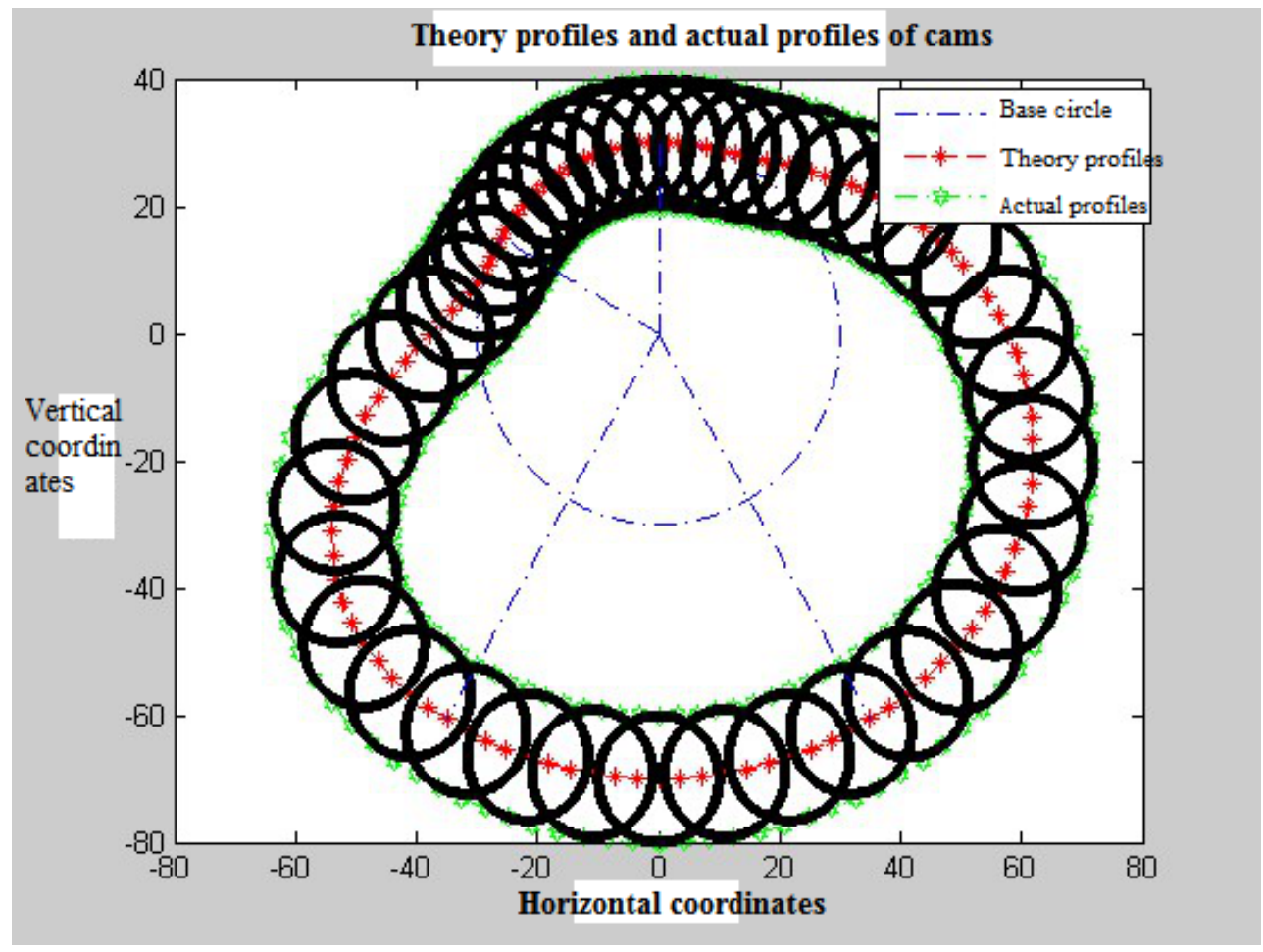

Fig. 2 Cam profile curve 


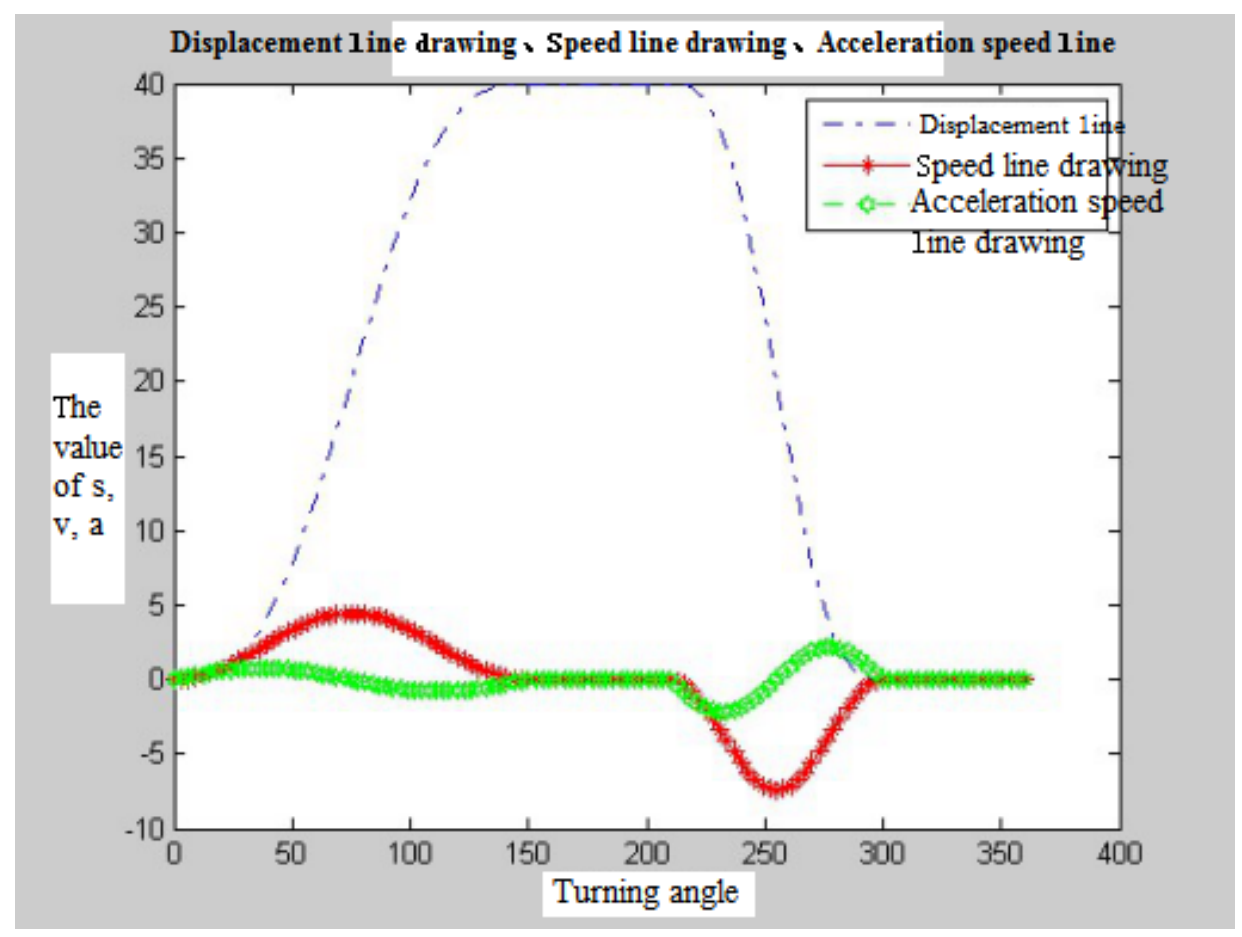

Fig. 3 Speed, displacement, and acceleration speed of the follower

After drawing the contour curve of the cam in MATLAB, click on the Desktop-workspace of the menu bar which then will appear the $\mathrm{M}$ double click, and the $\mathrm{X}, \mathrm{Y}$ coordinate value of the point on the curve can be put forward and saved as a. txt file after copying.

The contour design method is based on the example of the roller follower, and like the rotational center trajectory of a roller (theoretical contour), the movement track of the apex follower cam is the contour curve of the cam. While a flat-bottomed straight follower is similar to a biased apex follower, the programming method of the contour curve is similar in the case of the known movement law of the follower according to the curve equation and is no longer discussed.

\section{SolidWorks 3D Solid Modeling}

The design of mechanical products is not to design a separate part, but to make a plurality of parts an assembly body. In the product design, the design staff conceives its shape, structure design and overall assembly. With the development of three-dimensional CAD/CAM technology, the three-dimensional modeling technology of the product brings the engineering designers from the traditional two-dimensional design space to the three-dimensional design space, fundamentally changing the product design concept[4].

In the design and manufacture of the product, the description of the geometrical shape is the core part, which provides the basic parameters for the structure analysis and the process regulation. In the early design, the design thought provided by the designer was two-dimensional projection engineering drawing which failed to clearly and intuitively express the actual shape of the product and to foresee the the possible irrationality of product design, as well as to analyze the movement performance of the product's overall assembly mechanism. The design cycle is long as it needs to be repeatedly modified in the product development process. After the application of three-dimensional CAD technology, the designer can make three-dimensional modeling to the product directly, express the inside and outside structure of the product clearly, establish the virtual assembly body, carry on the finite element analysis, the optimization design, the simulation movement and the examination movement performance, promoting the transformation of the product design form.

\section{Cam model establishment}

The contour curve of the disc-shaped cam directly determines the movement of the follower in the mechanism. The contour curve is very important in the modeling. The curve is a non-circular curve, 
usually composed of several sections of the curve, while there is no curve shape about equation in the Solidworks, it's difficult to get accurate curve contour by relying on Solidworks in the sketch. Therefore, the cam modeling based on MATLAB has been designed on the basis of the cam curve profile.

The cam contour curve will be obtained in MATLAB, and the contour curve of cam can be generated by importing into SolidWorks[6]. Select the new part model in SolidWorks. Select the Datum plane, click "Insert"-"curve"-"curve through XYZ Point" in the toolbar of the interface, open the saved. txt file in the dialog box that appears, import the data, then generate the cam outline, as shown in Figure 4. Note that SolidWorks identifies three columns of data points without any tags, and before importing, three columns of data that are arranged as a whole in the. txt file into Excel do not appear x, Y, z, and then copied to Notepad, as shown in Figure 5. In addition, if the curve is not closed, you must modify the imported coordinate data to make the points on the curve coincide.

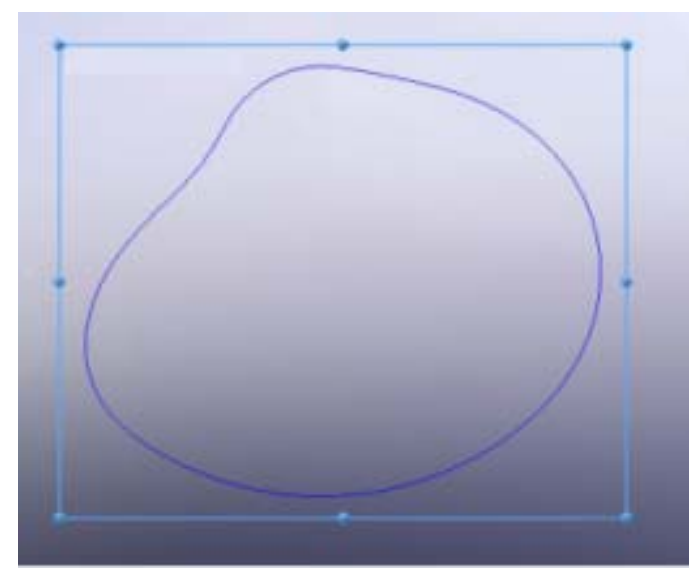

Fig. 4 Creating two-dimensional curve of cam profile

\begin{tabular}{|lll|}
\hline 0 & 25 & 0 \\
1.5869 & 19.9727 & 0 \\
2.2515 & 19.8917 & 0 \\
3.4909 & 19.76 & 0 \\
4.8023 & 19.5821 & 0 \\
6.1823 & 19.3639 & 0 \\
7.6271 & 19.1118 & 0 \\
9.1314 & 18.8322 & 0 \\
10.6896 & 18.5395 & 0 \\
12.2954 & 18.2105 & 0 \\
13.9428 & 17.8737 & 0 \\
15.6267 & 17.5187 & 0 \\
17.3436 & 17.1415 & 0 \\
19.5914 & 16.7354 & 0 \\
25.8691 & 16.2917 & 0 \\
22.6763 & 15.8502 & 0
\end{tabular}

Fig. 5 Cam curve Part data point

The curves generated in the interface can not be stretched to convert them to solid lines. Select the curve of the front datum, click on the "Insert"-"Sketch" selected curve, click the toolbar "Sketch Tools"-"conversion entity reference", the cam curve into a black solid line, the physical curve, You can get a 3D cam model, as shown in Figure 6. 


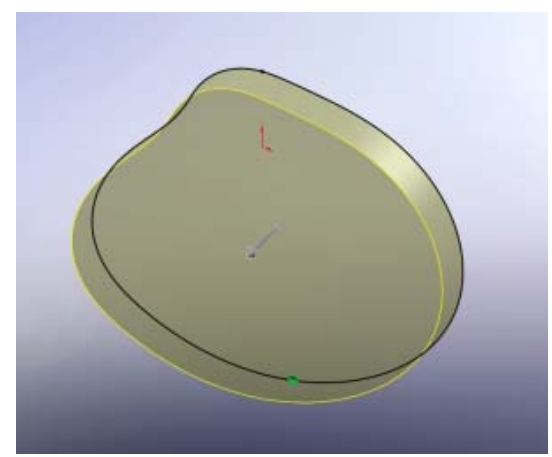

Fig. 6 The post-tension cam model

Based on the theoretical analysis of disc cam profile, the cam contour curve is fitted with MATLAB in this paper, which lays a solid foundation for the development of the SolidWorks by VB, and the parametric modeling of the cam can reduce the repetitive work in the design process, improve the design efficiency and shorten the product development cycle.

\section{References}

[1] LiXia, Liu Benxue,Zhang Sanchuan. The Design of Cam Contour Curve Based on MATLAB and Kinematics Simulation of Follower, Journal of Zhongyuan Institute of Technology, 2012, (1):41-43.

[2] Liu Xiongxin. Improvement of Kinetic Motion Law of Follower of CAM Mechanism, Packaging Engineering, 2010, 31 (10):63-65.

[3] Li Xinhua. Research and development of CAM mechanism CAD system, Huazhong Agriculture University, 2015.

[4] Muer. Matlab Practical Course, Electronic Industry Press, 2010:380-385.

[5] AutodeskCorp.AutodeskInventor[DB/OL].http://www.Autodesk.com.cn/adsk/servlet/ind.

[6] AutodeskCorp.AutodeskInventor[DB/OL].http://www.Autodesk.com.cn/adsk/servlet/ind.

[7] Xu Yuliang, Yue Zongfeng, Chen Jiadun. Two-time Development of CAM Design Module Based on SolidWorks, Modern machinery, 2012, (3):20-21.

[8] CAD/CAM/CAE Technology Alliance. From Green Hand to Master in Solidworks 2012, Tsinghua University Press, 2012. 